(Paper No. 4122.)

\title{
"A Method of determining the Initial Tensions in Rope Stays Supporting Towers or Similar Structures."
}

By William Edward Corrie, B.Sc., Assoc. M. Inst. C.E.

The method described in this communication was devised by the Author in order to facilitate the erection of the towers at a large wireless-telegraph station where he was acting as Resident Engineer; it depends on the measurement of the angle between a tangent to the rope and a horizontal line.

A rope stay may consist of a single piece or of several lengths connected by links. The stays on a radio-telegraphic station, for instance, are, as a rule, divided into sections which are linked together by insulators, the latter being inserted to prevent losses of power by the currents which would otherwise be induced in the wire rope. It is in general necessary therefore to allow for the weight and distribution of these insulators in addition to the weight of the rope itself. The stays consist of galvanized wire ropes of dimensions suited to the stress they have to bear.

Statement of the Problem.- The problem of calculating the initial tension it is desirable to put in a stay is simply the determination of the force which must be applied at a given point of the rope, and in a given tangential direction, in order to equilibrate a number of other forces, the magnitudes, points of application and directions of which are all known, and thus to maintain the stability of the structure. It is necessary to have some means of verifying whether the actual tension is equal to that so calculated.

The direction of the force is fixed by the measurement of the angle of a tangent to the stay to the horizontal at the point of application considered, which may conveniently be the lower end of the stay; the only unknown quantity is therefore the required tension.

Method of Solution.-After much preliminary calculation, and consideration of the fact that the curve in which the rope hangs 
is very approximately a catenary, the Author obtained a solution by the following method. The tension of the rope at the lower end may be found by taking moments of all the forces concerned about the upper end of the rope, and noting that for equilibrium of the guy the sum of these moments must be zero. In Fig. 1-

Let $\mathrm{T}_{0}=$ tension at the lower end of the guy;

$T_{1}=$ tension at the upper end;

$y=$ difference in height of the two ends ;

$x=$ horizontal distance between the two ends ;

$l=$ actual distance between the two ends (or a little less than the length of the guy if it were pulled out straight);

$\theta=$ angle of inclination of the guy rope, if it were straight, to the horizontal ;

or $\tan \theta=\frac{y}{x}$

$\psi=$ actual inclination of the guy rope to the horizontal at the lower end;

$\alpha=$ divergence of this inclination from the former $=\theta-\psi$;

$\mu=$ weight of guy rope per unit length ;

$\mathrm{W}=$ total weight of rope $=\mu . l$ very nearly;

$\mathrm{W}_{1}=$ weight of the link or insulator less the weight of the rope which it displaces, in the case where there is only one link. The deduction of the weight of rope displaced is merely for convenience in calculation, so that the rope itself may be regarded as continuous from end to end.

$l=$ distance of the centre of gravity of the link or links from the upper end along the guy.

Then the weight of the guy rope is $\mu . l$, plus a small amount due to the actual length being slightly greater than $l$, but in practice it will be found that any allowance for this will not materially affect the result in the case of a tight rope.

The angle $a$ depends on the tension required, and in the work under the supervision of the Author the greatest value was $5^{\circ} 40^{\prime}$ in the case of a rope, with five very heavy insulators, where the horizontal distance between the two ends was about double the difference in height. Except in a few special cases, such as the above, the value of the angle $a$ ranged from $1^{\circ}$ to $3^{\circ}$.

Guy ropes are usually drawn up fairly taut--that is, the sag in the rope is relatively small compared with the length, a fact which, in the Author's opinion, allows certain assumptions to be made 
without affecting the practical accuracy of the calculations. These assumptions are as follows :-

1. That the centre of gravity of the guy rope is on a vertical line midway between the two ends of the rope.

2. That a short length of the guy rope, say up to $1 \frac{1}{2}$ per cent. of the total length, is, for all practical purposes, straight.

\section{Fig. 1.}

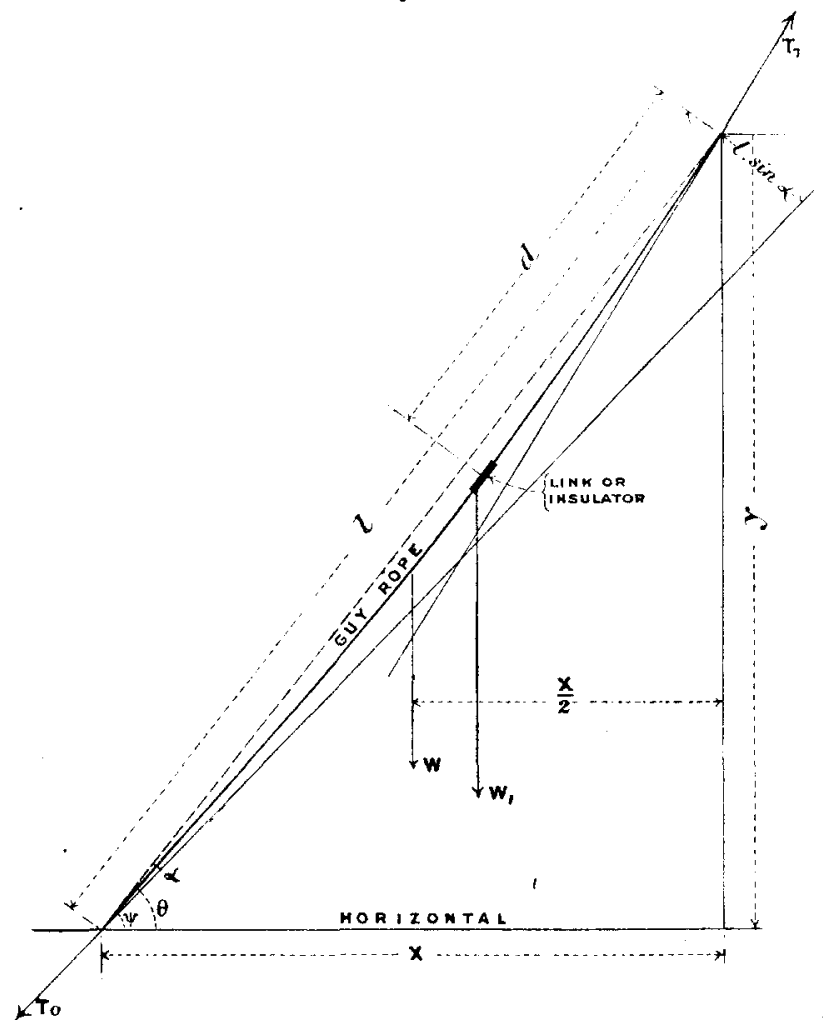

3. That, in the case of a guy rope composed of several pieces connected by links, such as insulators, the borizontal distance of the centre of gravity of any one link, from either end, may be calculated as if the guy were straight between the two ends, or, if a nearer approach to accuracy be required, an allowance may be made, depending on the angle of inclination of the guy to the horizontal. 
The Method in Practice.-As already pointed out, the tension at the lower end of the rope may be found by taking moments about the upper end. As will be seen from Fig. 1, the perpendicular distance of the upper end from the direction of the tension at the lower end is $l \cdot \sin a$. Hence the moment of the tension at the lower end, $\mathrm{T}_{0}$, about the upper end, is therefore $\mathrm{T}_{0} \cdot l \cdot \sin a$.

The moment of the weight of the rope about the upper end plus the moment of the weight of the link (less the weight of the rope displaced) about the upper end is

$$
\mu . l \cdot \frac{x}{2}+\mathrm{W}_{1} d \cos (\theta+\alpha / 2) .
$$

[The angle in the second term of the above is assumed to be $(\theta+a / 2)$, which is very close, as the true value is obviously between $\theta$ and $(\theta+\alpha)$ and $\alpha$ is a very small angle.]

Hence equating moments

or

$$
\mathrm{T}_{0} \cdot l \cdot \sin a=\mu \cdot l \cdot \frac{x}{2}+\mathrm{W}_{1} d \cos (\theta+a / 2)
$$

$$
\mathrm{T}_{0}=\frac{1}{l}\left[\mu \cdot l \cdot \frac{x}{2}+\mathrm{W}_{1} d \cos (\theta+\alpha / 2)\right] \operatorname{cosec} \alpha .
$$

All the quantities on the right-hand side of the above equation are known. Hence it may be written as

$$
\mathrm{T}_{0}=\mathrm{K} \operatorname{cosec} \alpha,
$$

where $\mathbf{K}$ is approximately constant for a given guy.

The actual tension in the guy rope can be obtained from this expression by measuring the angle $a$, so long as there is no great departure from the required tension.

To facilitate practical use the equation $T_{0}=K \operatorname{cosec} a$ can be plotted as a curve, with the tensions as ordinates and corresponding values of $\operatorname{cosec} \alpha$ as abscissas. For this purpose the value of $\alpha$ should be assumed, either from previous experience, or by experiment in a given case, in order to calculate $(\theta+a / 2)$, and thus obtain $\mathrm{K}$ very approximately. It may be found later that the value of a taken was not quite correct, but this can easily be rectified, as also the addition of $\alpha / 2$ to $\theta$, which possibly should have been some other fraction between $0.4 \alpha$ and $0.6 \alpha$; but it is questionable if such refinement is necessary.

Towers Erected.-The work under the direct supervision of the Author included six square-framed timber towers 300 feet high, 
each supported by six sets of four guys in each set, and one triangular-framed steel tower 500 feet high, supported by two sets of three guys each. The number of guy ropes erected was 150 , all of steel-wire rope, with from one to six links or insulators in each. All the timber towers were precisely alike, except that the slope of the ground, and therefore the level of each anchorage, varied slightly. The Author made calculations for a tower on level ground, and found, after making further calculations for a few special cases, that, even if the slope of the ground was about 1 in 40 , there was no practical difference whether the anchorage was above or below the level of the tower-foundation.

Therefore the angle a for any one set of four guys on any timber tower is the same for the corresponding set on any other timber

Fig. 2.

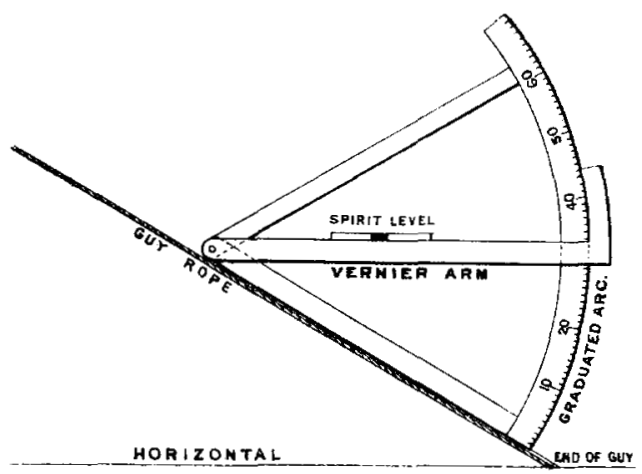

tower. Hence, for six towers, with six sets of four guys each, there were twenty-four guys with the same angle $\alpha$. The angle $\theta$ must, however, be calculated for each single guy.

An instrument is required for the measurement of the angle of inclination of the guy, and a suitable arrangement consists of a sector of a circle graduated from $0^{\circ}$ to, say, $60^{\circ}$ along the circumference to half degrees. A vernier arm, on which is mounted a spirit-level, is fitted to swing round the centre of the circle, as shown in Fig. 2. The instrument is adjusted so that the reading is zero when one radius of the sector is horizontal. Then the sector is placed on the guy, and the vernier arm moved to the horizontal, shown by the spirit-level on the arm. The angle read on the instrument is the tangential inclination of the guy to the horizontal, or the angle $\psi$; then, as previously shown, $\alpha=\theta-\psi$. 
Example of Determination of Tension.--The following example is given of the top guy of a 300 -foot timber tower.

- The rope was $1 \frac{1}{2}$ inch diameter steel rope, weighing 3.55 lbs. per foot length, and there were five intermediate links or insulators. It was found that the moment of the weight of rope was 229,000 Ibs.-feet, and that of the weight of insulators was 151,900 lbs.-feet. The sum is $=380,900$ lbs.-feet.

The length of the guy was $426 \cdot 35$ feet, and the required initial

Fig. 3.

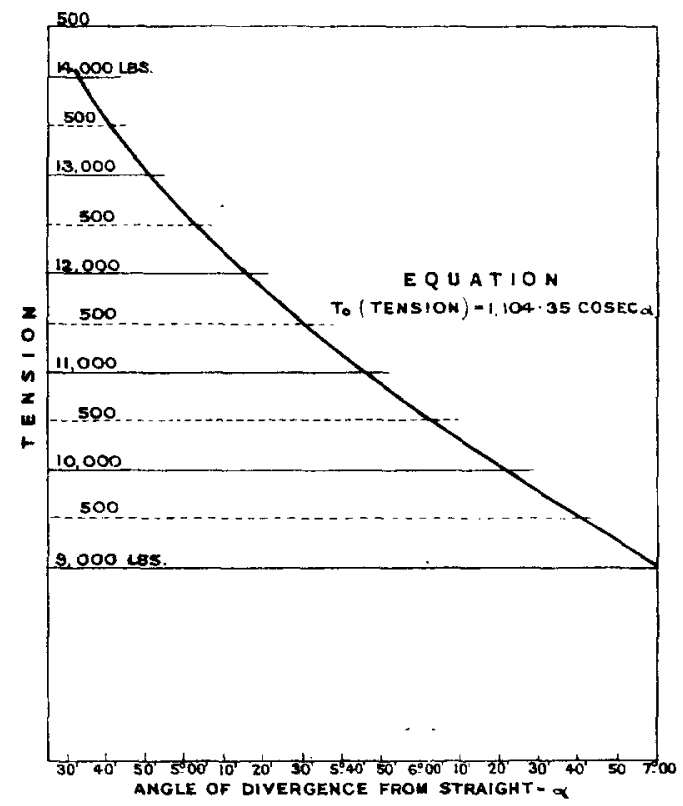

tension was 8 tons, or 17,920 lbs., and $\alpha$ was measured by the instrument already described, and was found to be $3^{\circ}$.

$K$, in the equation $T_{0}=K \operatorname{cosec} \alpha$, is $\frac{380,900}{426 \cdot 35}=893$.

Hence $\mathrm{T}_{0}=893 \operatorname{cosec} \alpha$.

Substituting $\mathrm{T}_{0}=17,920 \mathrm{lbs}$, it is found that $\alpha=2^{\circ} 51^{\prime} 30^{\prime \prime}$, which is near enough to $3^{\circ}$ to make no practical difference, or, in other words, the actual tension was approximately that desired.

The equation $\mathbf{T}_{0}=893$ cosec $a$ was plotted as a curve, and was used for the four top guys of the six timber towers.

The errors caused by the various assumptions made are shown as follows :- 
Limits of Possible Errors.--The length of rope being a few inches greater than that taken, let it be supposed that the weight is increased 10 lbs., which is obviously far beyond the mark. Let it be supposed also that the centre of gravity of the insulators is 4 feet further horizontally from the tower than originally taken, obviously a far greater allowance for inaccuracy than necessary.

Then it was found that the sum of the moments of the guy and the insulators was 384,700 lbs.-feet, so that

$$
\begin{aligned}
\mathrm{T}_{1} & =\frac{384,700}{426 \cdot 35} \operatorname{cosec} \alpha \\
& =901 \cdot 5 \operatorname{cosec} \alpha .
\end{aligned}
$$

Hence for $\alpha=2^{\circ} 51^{\prime} 30^{\prime \prime} \quad \mathrm{T}_{0}=18,095 \mathrm{lbs}$, or $175 \mathrm{lbs}$. more than the former result, a difference of less than 1 per cent.

The errors introduced by the ground not being level, resulting in each anchorage being above or below the level of the foundation of the tower, are shown by considering an extreme case where the anchorage was nearly 16 feet below the tower foundation.

The length, $l=437 \cdot 7$ feet, and the sum of the moments of the weight of rope and of the insulator was $=382,700 \mathrm{lbs}$.-feet, so that

$$
\begin{aligned}
\mathbf{T}_{0} & =\frac{382,700}{437 \cdot 7} \operatorname{cosec} \alpha \\
& =874 \operatorname{cosec} \alpha .
\end{aligned}
$$

Hence for $a=2^{\circ} 5 \mathrm{l}^{\prime} 30^{\prime \prime} \mathrm{T}_{0}=17,540 \mathrm{lbs}$, , or $380 \mathrm{lbs}$. less than the former result of $17,920 \mathrm{lbs}$., or $2 \cdot 12$ per cent.

All the lower guys were of lighter rope, namely, $\frac{7}{8}$ inch, with fewer and lighter links or insulators, and the errors were found to be much smaller.

The diagram in Fig. 3 is that for the lower guys of the 500 -foot steel tower, and represents the extreme case of the greatest value of the angle of divergence $\alpha$.

The Author was the Resident Engineer for the construction of the towers on the large radio-telegraphic station, for which the firm of Messrs. Clark, Forde, Taylor and Erskine-Murray were Consulting Engineers, and he is indebted to them for permission to present this Paper, and especially to Dr. J. Erskine-Murray for many helpful suggestions in its preparation.

The Paper is accompanied by three sheets of drawings, from which the Figures in the text have been prepared. 